AperTO - Archivio Istituzionale Open Access dell'Università di Torino

\title{
Assessment of the Precision ID Identity Panel kit on challenging forensic samples
}

\section{This is a pre print version of the following article:}

Original Citation:

\section{Availability:}

This version is available http://hdl.handle.net/2318/1761367

since 2021-07-08T13:34:50Z

Published version:

DOI:10.1016/j.fsigen.2020.102400

Terms of use:

Open Access

Anyone can freely access the full text of works made available as "Open Access". Works made available under a Creative Commons license can be used according to the terms and conditions of said license. Use of all other works requires consent of the right holder (author or publisher) if not exempted from copyright protection by the applicable law. 


\section{Assessment of the Precision ID Identity Panel kit on challenging forensic samples}

2

3 Chiara Turchi ${ }^{1}$, Carlo Previderè ${ }^{2}$, Carla Bini ${ }^{3}$, Eugenia Carnevali ${ }^{4}$, Pierangela Grignani ${ }^{2}$, Alessandro 4 Manfredi $^{5}$, Filomena Melchionda ${ }^{1}$, Valerio Onofri ${ }^{1}$, Susi Pelotti ${ }^{3}$, Carlo Robino ${ }^{6}$, Solange Sorçaburu5 Ciglieri $^{5}$, Adriano Tagliabracci ${ }^{1}$, Paolo Fattorini ${ }^{5 \S}$

6

7

2

3

4

${ }^{5}$ Department of Medicine, Surgery and Health, University of Trieste, Italy

6

${ }^{\S}$ Corresponding author

8

9

10

11

12

13

14

15

16 


\section{Highlights}

2

3 The precision of the ID Identity Panel kit was assessed on a large set of challenging forensic

4 samples.

5

6 A threshold of 50 reads for locus call reduces the frequency of sequencing errors.

7

8 Replicate analyses assure a low/null rate of typing errors.

9

10 The high number of markers of the kit assures a random match of probability $\leq 1.6 \times 10^{-13}$ even for 11 the most challenging samples.

12

PCR-MPS of SNP markers is the ideal approach to the analysis of LCN and degraded DNAs. 


\section{Abstract} The performance of the Precision ID Identity Panel (Thermofisher) was assessed on a set of 87 forensic samples with different levels of degradation for which a reference sample from the "same donor" or from a "first degree relative" was available. PCR-MPS analysis was performed with DNA input ranging from $1 \mathrm{ng}$ to $12 \mathrm{pg}$ and through 21-26 PCR cycles, in replicate tests, and a total number of 255 libraries were sequenced on the Ion Personal Genome Machine ${ }^{\mathrm{TM}}$ (PGM ${ }^{\mathrm{TM}}$ ) System. The evaluation of the molecular data allowed to set a fix threshold for locus call at $50 \mathrm{x}$ which suitably worked even when low amounts of degraded DNA (12 pg) were investigated. In these analytical conditions, in fact, 25 PCR cycles allowed the genotyping of about $50 \%$ and $35 \%$ of the autosomal and the Y-specific markers on average, respectively, for each single amplification with a negligible frequency of drop ins $(0.01 \%)$. On the other hand, drop out artefacts reached 18-23\% when low copy number and degraded DNA samples were studied, with surviving alleles showing more than 600 reads in $2.9 \%$ of the cases. Our data pointed out that the Precision ID Identity Panel allowed accurate typing of almost any amount of good quality/moderately degraded DNA samples, in duplicate tests. The analysis of low copy number DNAs evidenced that the same allele of a heterozygous genotype could be lost twice, thus suggesting that a third amplification could be useful for a correct genotype assignment in these peculiar cases. Using the consensus approach, a limited number of genotyping errors were computed and about $37 \%$ of the autosomal markers was finally typed with a corresponding combined random match probability of at least $1.6 \times 10^{-13}$, which can be considered an excellent result for this kind of challenging samples. In the end, the results presented in this study emphasize the crucial role of the expert opinion in the correct evaluation of artefacts arising from PCR-MPS technology that could potentially lead to genetic mistyping. (3) 


\section{Introduction}

The current golden standard for DNA-based human identification is multiplex PCR followed by capillary electrophoresis of the amplified products (PCR-CE) [1,2]. Short tandem repeats (STRs) are the selected markers for genotyping a wide range of forensic specimens even if markers such as SNPs may be used in other scenarios [3]. In particular, the molecular structure of the SNPs (which, in case of bi-allelic SNPs, is based either on a transition or a transversion of the wild type nucleotide) coupled with the reduced molecular size of the amplicons makes this class of markers the best choice for the genetic identification of degraded samples [4,5]. It is also true, however, that PCR-CE may not perform efficiently when low copy number and/or very degraded DNA is recovered from the samples [1-3]. In these cases, in fact, even if several analytical approaches have been developed in the last two decades, the resulting genetic profiles can be of scarce utility or even inconclusive [1-3,6,7].

More recently, Massive Parallel Sequencing (MPS) technologies also entered the field of Forensic Genetics [8], allowing the customization of several kits. Even if MPS relies on PCR technology as well, the most important advantage over the conventional PCR-CE approach is that a huge number of markers with different molecular structure (e.g., STR and SNP) can be simultaneously multiplexed $[8,9]$.

A few years ago, ThermoFisher company tested a new PCR-MPS panel, the HID-Ion AmpliSeq ${ }^{\mathrm{TM}}$ Identity Panel kit, which allowed the genetic characterization of 103 autosomal SNPs plus 33 Ymarkers [10]. After optimization of the prototypes [11,12], the ultimate kit format now allows the simultaneous typing of 90 and 34 autosomal and Y-specific SNPs, respectively. The performances of this kit have been tested on control and reference samples (e.g., fresh samples) in several studies [1323]; the resulting combined random match probabilities calculated in different populations are between $1 \times 10^{-34}$ and $1 \times 10^{-37}$ [16-21,24,25]. However, only few studies focused on degraded samples, that is samples whose analysis often shows partial or inconclusive genetic results. In particular, Getting et al. [26] described the advantage of the use of this kit over conventional PCR-CE commercial panels on a set of selected fragments eluted from sonicated DNA samples. More recently, Salata et al. [27] reported the performances of this kit on a set of artificially degraded samples, among which a set of heat-treated bloodstains, and on a set of quite homogeneous forensic specimens (ten bones and one tooth). Avila et al. [28] increased the set of forensic samples to 47 criminal specimens whose degradation indexes (DI) were however very similar to the ones of unmodified control samples (DI < 1.5). Therefore, the performance of this kit should be investigated on more challenging degraded/damaged real casework samples. It is worth mentioning that in two [27,28] out of these three studies no threshold value was fixed for the locus call and a default coverage value of $6 \mathrm{x}$ was adopted for genotyping. The definition of calling thresholds has been questioned from the very 
beginning of the PCR era $[8,12]$, but currently the shared opinion is that the absence of threshold values for a locus call would lead to unreliable results since background noise signals could be erroneously mistaken as true alleles $[8,12,14,15]$.

The aim of the present study is to assess the performance of the HID-Ion AmpliSeq ${ }^{\mathrm{TM}}$ Identity Panel kit on the $\mathrm{PGM}^{\mathrm{TM}}$ platform starting from sub-optimal amounts (down to $12 \mathrm{pg}$ ) of DNA recovered from degraded/damaged real casework forensic samples. In addition, the analytical condition leading to a reliable locus call will be evaluated.

\section{Material and methods}

\subsection{Ethic statement}

This study was approved by the Ethical Comity of the University of Trieste, Italy (101/04.12.2019). Written informed consent was obtained from all living donors involved in the study.

\subsection{DNA Samples}

Five laboratories collected a set of 87 forensic samples including saliva swabs $(n=20)$, bone remains $(n=19)$, cadaveric blood $(n=7)$, cadaveric muscle $(n=3)$, fingernails $(n=2)$, FFPE tissues $(n=15)$ and touch DNAs $(\mathrm{n}=16)$. The aging of the samples together with other relevant information on the samples are reported in Table S1. The availability of a reference sample (either "same donor" sample or "first degree relative" sample) was the recruitment criterion. In addition, DNA sample FM, already employed in other PCR-MPS studies [29], was used to produce a set of artificially degraded DNAs $(\mathrm{n}=5)$ following published protocols [30].

DNA quantification was performed by a single selected lab which was provided with six-ten microliters aliquots of each sample in dry ice. DNA was quantified by duplicated tests on the Applied Biosystems ${ }^{\circledR} 7500$ real-time PCR System (Thermo Fisher Scientific) using the Quantifiler ${ }^{\mathrm{TM}}$ Trio DNA Quantification Kit (Thermo Fisher Scientific). In agreement with ref [27], when the amount of sample was below the Limit of Quantification $(0.005 \mathrm{ng} / \mu \mathrm{l})$, as assessed by the long target, the degradation index (DI) was stated as "not computable" (nc). According to their DI, the samples were pooled into three categories which were arbitrarily designed as follows: DI < 5 (not degraded/moderately degraded), DI > 9 (degraded) and DI nc (very degraded), respectively. The samples were finally sequenced in a selected laboratory. For sensitivity studies, the 2800M DNA (Promega) was diluted appropriately. 


\subsection{Library construction, template preparation, enrichment and MPS sequencing}

Two hundred-fifty-five libraries were prepared by using the Precision ID Identity Panel and the Precision ID Library Kit (Thermo Fisher Scientific) according to the user guide [31]. Amplifications of genomic DNA target were performed with DNA input ranging from $1 \mathrm{ng}$ to $12 \mathrm{pg}$ and through a different number of PCR cycles (from 21 to 26), as outlined in Table S2. At least one negative amplification (no template) control was added in each round of PCR.

The total library-PCR system volume was $20 \mu \mathrm{L}$, which consisted of $4 \mu \mathrm{L}$ of $5 \mathrm{X}$ Ion AmpliSeq ${ }^{\mathrm{TM}}$ HiFi Mix, $10 \mu \mathrm{L}$ of Precision ID Identity Panel primer pool, DNA and nuclease-free water as needed to reach the total volume for each specific experiment. Thermal cycling was performed on the

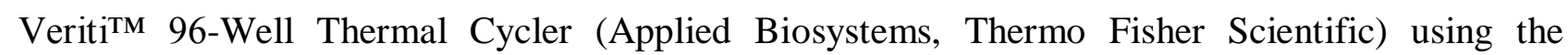
following conditions: enzyme activation for $2 \mathrm{~min}$ at $99^{\circ} \mathrm{C}$; denaturation, annealing and extension for $21-26$ cycles of $15 \mathrm{~s}$ at $99^{\circ} \mathrm{C}$ and 4 min at $60^{\circ} \mathrm{C}$. After PCR, the amplicons were partially digested by adding $2 \mu \mathrm{L}$ FuPa Reagent (Thermo Fisher Scientific) and incubated for $10 \mathrm{~min}$ at $50^{\circ} \mathrm{C}$, for $10 \mathrm{~min}$ at $55^{\circ} \mathrm{C}$, and for $20 \mathrm{~min}$ at $60^{\circ} \mathrm{C}$. Ligation of barcode adapters was performed by adding $4 \mu \mathrm{L}$ Switch Solution, $2 \mu \mathrm{L}$ DNA Ligase and $2 \mu \mathrm{L}$ diluted Ion Xpress ${ }^{\mathrm{TM}}$ Barcode Adapters into $22 \mu \mathrm{L}$ digested PCR reaction, and followed by incubation for $30 \mathrm{~min}$ at $22^{\circ} \mathrm{C}$ and $10 \mathrm{~min}$ at $72^{\circ} \mathrm{C}$. A negative control of ligation was also carried out by adding $4 \mu \mathrm{L}$ Switch Solution, $2 \mu \mathrm{L}$ DNA Ligase and $2 \mu \mathrm{L}$ diluted IonXpress_063 Barcode Adapters into $22 \mu \mathrm{L}$ of water. After ligation, each library was purified with Agencourt $^{\mathrm{TM}}$ AMPure ${ }^{\mathrm{TM}}$ XP Reagent (Beckman Coulter) and then quantified by Ion Library TaqMan $^{\text {TM }}$ Quantitation Kit (Thermo Fisher Scientific) [31,32].

Six libraries (whose concentration ranged from 6.7 to $16.6 \mathrm{pM}$ ) were submitted to the optional step [31] of library amplification with $72 \mu \mathrm{L}$ Platinum $^{\mathrm{TM}}$ PCR SuperMix HiFi and $3 \mu \mathrm{L}$ Library Amplification Primer Mix using the following conditions: 2 min at $98^{\circ} \mathrm{C}, 8$ cycles of $15 \mathrm{~s}$ at $98^{\circ} \mathrm{C}$ and $1 \mathrm{~min}$ at $64^{\circ} \mathrm{C}$. The amplified libraries were purified with Agencourt ${ }^{\mathrm{TM}}$ AMPure ${ }^{\mathrm{TM}}$ XP Reagent (Beckman Coulter) and then quantified as reported above.

All the libraries were diluted to $8 \mathrm{pM}$, pooled in equivolume and then submitted to emulsion PCR to generate template positive Ion Sphere ${ }^{\mathrm{TM}}$ Particle (ISPs) containing clonally amplified DNA. Emulsion PCR (emPCR) was performed on the Ion OneTouch ${ }^{\mathrm{TM}} 2$ Instrument (Thermo Fisher Scientific) with the Ion PGM ${ }^{\mathrm{TM}} \mathrm{Hi}^{-} \mathrm{Q}^{\mathrm{TM}}$ View OT2 Kit (Thermo Fisher Scientific). Templatepositive ISPs were enriched with the Ion OneTouch ${ }^{\mathrm{TM}}$ ES (Thermo Fisher Scientific) following the manufacturer's recommendations. Sequencing was performed on the Ion Personal Genome Machine ${ }^{\mathrm{TM}}\left(\mathrm{PGM}^{\mathrm{TM}}\right)$ System using the Ion PGM ${ }^{\mathrm{TM}} \mathrm{Hi}^{-} \mathrm{Q}^{\mathrm{TM}}$ View Sequencing Kit in 200-base read mode. Sequencing primer and Control Ion Sphere ${ }^{\mathrm{TM}}$ Particles were added to the enriched, template- 
positive ISPs. After the sequencing primer annealing, a sequencing polymerase was added and a final volume of $30 \mu \mathrm{L}$ was loaded onto Ion $318^{\mathrm{TM}}$ Chip v2 (Thermo Fisher Scientific). Three libraries (namely \#256, \#257 and \#258) were used in the fourth chip without dilution to $8 \mathrm{pM}$. In total, 255 barcoded libraries were sequenced on four Ion $318^{\mathrm{TM}}$ Chip v2, as outlined in Tables S2.

\subsection{Sequencing data analysis}

The alignment of reads against Homo Sapiens reference genome (GRCh37/hg19) was performed on the Torrent Suite ${ }^{\mathrm{TM}}$ v5.0.4 server. Coverage analysis was carried out by CoverageAnalysis v5.0.4.0 plugin [33], which provided statistics and graphs describing the level of sequence coverage produced for Precision ID Identity targeted regions. Information about mapped reads, on-target percentage and mean depth of coverage were downloaded for each sample library (Barcode Summary Report file). Individual SNP genotypes were called by the HID_SNP_Genotyper v4.3.2 plugin with DNA target regions identified by the PrecisionID_IdentityPanel_targets.bed (Amplicon_Insert_IISNPv3) and known SNP variants listed in PrecisionID_IdentityPanel_hotspots.bed (iiSNP_FP_v2_hotspot) files. These resulting excel files were then used for data analysis.

Eighteen libraries related to ten different bone samples which showed above $10 \%$ of unmapped reads (range 13\% - 82\%; mean 43\%) were submitted to further analysis in order to verify the occurrence of out of target DNA amplification and sequencing. In these samples, unmapped reads were filtered by using the "BAM filter" tool on Galaxy [34]. The derivative unmapped .bam files were then analyzed on FastQC version 0.11.8 [35] in order to check the presence of overrepresented reads. They were then downloaded and analyzed by Clustal W version 2.1 (Larkin, M.A 2007). The output of the sequence alignment was visualized by Jalview 2.11.0 and the most represented sequences were compared on BLAST to finds regions of similarity between biological sequences.

\subsection{Data analysis}

According to the DNA input used for PCR, the data of the selected libraries were pooled into three categories that were named as follows: OTQ (Optimal Template Quantity, e.g., 0.1-1.0 ng), STQ (Sub-optimal Template Quantity, e.g., 13-99 pg) and MTQ (Minimal Template Quantity, e.g., 12 pg). In addition, each group was further divided in three categories according to the degradation index (DI) of the samples. Therefore, nine classes of samples were in total defined (for example, the class OTQ/nc includes samples amplified with an amount of DNA from 0.1 to $1.0 \mathrm{ng}$ and with degradation index not computable). 
1 The relative Depth of Coverage $(\mathrm{rDoC})$ was calculated as the ratio between the coverage for a specific

2 SNP locus and the overall coverage of the sample [37]. Comparison between the rDoC values was

3 performed by $r^{2}$ test. Noise was calculated as reported elsewhere $[14,15]$.

4 The presence of any of the four flags provided by the software (Major Allele Frequency, No Locus

5 Call, Percentage of Positive Coverage and Coverage) was scored and used for further elaboration.

6 Any replicate data were averaged.

7

8

\subsection{Genotyping}

The minimum coverage for locus call was empirically fixed at $50 \mathrm{x}$ (see paragraph 3.6). All the genotyping data were used irrespective both of their GQ (Genotype Quality) and the presence of flags. The correctness of the results was checked by two operators visually. In particular, the results of each test were compared with those of the corresponding reference samples and/or with those of the tests performed with higher amounts of the same sample.

The frequency of amplification artefacts (both allelic drop outs and allelic drop ins) as well as the frequency of flags were given as the ratio between the total number of this kind of events out of the total number of markers with at least 50 reads of coverage.

\subsection{Consensus genotype}

Standard methods $(>(\mathrm{n} / 2)+1)$ [38] were used to build the consensus genotype by using the data of two replicates. When analyzing the data of three replicates, a modification of the method proposed by [27] was used. In particular, the homozygosity was assigned only if each of the three replicates gave the same homozygous pattern. The heterozygosity $(\mathrm{Ht})$ was assigned in the following four cases: 1) three identical heterozygous genotypes; 2) two identical heterozygous and one homozygous genotypes (in agreement with allelic drop out); 3) two identical heterozygous and one undetermined genotypes (for example $<50 \mathrm{x}$ ); 4) one heterozygous and two homozygous genotypes for different alleles (both in agreement with allelic drop outs). In case of one heterozygous plus two identical homozygous genotypes (for example, $\mathrm{A} / \mathrm{G}+\mathrm{A} / \mathrm{A}+\mathrm{A} / \mathrm{A}$ ), no result was assigned.

\subsection{Calculations and graphs}

Microsoft Excel 2007 and Stata/SE version 12.1 (StataCorp) were used for calculations and graphs. For statistical analyses ( $t$-test and ANOVA, when appropriate) significance was assumed with $p$ values $<0.05$. 


\section{Results and discussion}

\subsection{DNA quantification}

The samples were quantified in duplicate experiments using the QuantifilerTM Trio DNA Quantification Kit (Thermo Fisher Scientific). Quantifications were scored according to the Small Autosomal (SA) Probe and reported in Table S1 together with the mean of the corresponding values. In total, 59 samples showed a DI < 5 (average: 1.7; sd; 1.0; median: 1.3), 9 samples showed a DI > 9 (average: 24; sd; 35; median: 24; max: 110), while 19 samples showed a not computable DI. No sample showed PCR inhibition as shown by the Ct values $(<26)$ of the Internal PCR Control (IPC) probe of the kit.

\subsection{The sequencing data: a general view}

The main parameters of the four Ion 318 Chip run in this study are reported in Table S3. Out of the 11,287,275 addressable wells, $63.3 \%$ showed Ion Sphere ${ }^{\mathrm{TM}}$ Particles (ISPs) in average, with more than $98.0 \%$ of the ISPs represented by the libraries. The final ISPs libraries were 3,462,471 (48.9\% of the total). These results fulfilled the recommended parameters for Ion $318^{\mathrm{TM}}$ Chip v2 [31,33]. The percentage of low quality and adapter dimer sequences was however high (up to $17.7 \%$ and $15.7 \%$, respectively) in the chips loaded with libraries built with low amounts of template [12].

Table 1 shows the values of library concentration (1.c.), mapped reads (m.r.), mean depth of coverage (m.d.c.) and percentage of on-target reads (\% o-t.r.) assessed for 193 out of 255 libraries subdivided according to each quantitative category based on the amount of DNA input (the data of the remaining 62 libraries were used for comparison and/or other purposes (see Table S4)). The results show a progressive decay of the values for all these parameters directly related to the decrease of the DNA amount used for the amplification steps $\left(p\right.$-value $\left.\leq 4.9 \times 10^{-4}\right)$. Figure S1 shows how the different numbers of PCR cycles affected the picomolarity of the libraries whose concentration was directly related to the number of PCR cycles (for OTQ from the $24^{\text {th }}$ cycle and for the other two categories for the $26^{\text {th }}$ cycle; $p$-value $\leq 1.0 \times 10^{-5}$ ) [27]. Even if the pM of the MTQ libraries were higher than the no-template control ( $p$-value $=0.0007$ ), almost half of the MTQ libraries (23 out of 51) showed concentration values below $17.9 \mathrm{pM}$ (that is, in the range of the mean value plus one standard deviation of the no-template controls). Therefore, it is likely that the accuracy in diluting the MTQ libraries down to $8 \mathrm{pM}$ was poor. This provides an explanation for the low m.r. and m.d.c. values found for 12 pg DNA samples amplified through 22-24 PCR cycles, as well as for the outliers of Figures S2 and S3. 


\subsection{Blank controls}

A total number of 17 no-template controls were run together with the samples. The mean value and standard deviation of the libraries' concentration was 10.7 \pm 7.2 (median value was 9.3; min= 2.0; $\max =28.4$ ), while the other sequencing parameters are shown in Table 1. In average, 0.5 markers per samples showed a coverage higher than 50 reads, with the exception of library \#106. In that case, 14 markers showed a coverage $>50 \mathrm{x}$, consistent with a contamination occurred during library preparation.

The duplicate analysis of the negative control set up during the extraction of a set of FFPE samples showed a minimal contamination (with 8 markers above $50 \mathrm{x}$ ) consistent with the following finding of an excess of drop-ins in those samples (see paragraph 3.11).

\subsection{Noise}

Ten positive control samples (DNA from the $2800 \mathrm{M}$ cell line), amplified for 21-26 cycles with DNA amounts varying from 0.1 to $1 \mathrm{ng}$, were used for this calculation. The mean noise ratio for the autosomal and Y markers was $0.054 \%$ and $0.038 \%$, respectively. In addition, the noise ratio was determined in 29 samples showing DI $<5$, amplified for 21 PCR cycles starting from $1 \mathrm{ng}$ of DNA template. The noise ratio was below $0.083 \%$ in all samples. The mean male noise ratio in 17 female samples out of the 29 described above, calculated as the number of Y-SNPs reads out of the total number of reads, was $0.038 \%$.

Overall, the results showed that the noise ratio in the samples analyzed in this study is very low, even lower than the value found by $[13,14]$. Four single samples exhibited a remarkable high noise ratio (until 11\%) just for a very limited number of markers (which were however flagged by the software), which were probably originated by contamination issues [14].

Finally, the noise ratio was tested even in 0.27-1 ng of nine degraded samples amplified through 2126 cycles of PCR. The results were similar to the values recorded for the samples described above $(p$-value $>0.065)$.

\subsection{Locus balance}

The locus balance among autosomal and Y-specific loci was evaluated by a parameter defined relative depth of coverage (rDOC), which is the ratio between the coverage of each locus and the overall coverage of the sample [37].

Twenty-six DNA samples with a DI $<5$, amplified starting from $1 \mathrm{ng}$, and nine tests performed with 0.1-1 ng of the $2800 \mathrm{M}$ DNA were selected to this aim. The autosomal loci were covered by $84.1 \%$ and $84.2 \%$ of the reads of the two groups of samples, respectively, in agreement with the data of ref. 
1 [13-15,27]. In addition, the mean rDOC of these two sets of samples was compared showing a good 2 coefficient of correlation $r^{2}=0.810$. Figures S4A and S4B show the $\mathrm{rDoC}$ of the 26 samples with a

3 DI $<5$. Overall, as previously described [13-15], the locus balance did not correlate with the molecular weight of the amplicons, but it rather reflected the molecular features of the primer sets designed for the multiplex PCR.

In order to investigate the locus balance in degraded samples, five DNAs (namely S2, S21, S17, S40 e S43) with degradation indexes between 5 and nc were selected. The mean $\mathrm{rDOC}$ for each marker resulting from the analysis of $1 \mathrm{ng}$ DNA was then compared to the one obtained from the $2800 \mathrm{M}$ DNA samples. The results for the autosomal and Y-specific SNPs comparison are described in Figures S5A and S5B, respectively. The data show that, in degraded DNA samples, the rDoC depends mainly on the molecular weight of the amplicons, as low molecular weight loci were preferentially amplified. The locus balance of the depurinated DNA samples showed a peculiar trend, different from the one of the five "naturally" degraded samples described above as six autosomal (rs2292972, rs576261, rs719366, rs430046, rs221956 e rs873196) and one Y-specific markers (rs372157627) showed higher DoC than those of the $2800 \mathrm{M}$ control DNAs. The finding that a set of loci exhibited a peculiar behaviour in PCR/CE [30] and PCR/MPS analysis of depurinated DNA samples has been already described elsewhere [27,29] and can be ascribed to the peculiar damaging pattern affecting the purine nucleobases in the DNA sequence.

Finally, to assess the intra and inter repeatability of the experiments, the rDOC of duplicate tests were compared both for the $2800 \mathrm{M}$ control DNA and the five above mentioned degraded samples. The coefficient of correlation remained good $\left(r^{2}>0.658\right)$ for amounts of template corresponding to 270 $300 \mathrm{pg}$ while it significantly decreased when $12 \mathrm{pg}$ of DNA were used $\left(r^{2}<-0.004\right)$. In conclusion, the repeatibility of the $\mathrm{rDoC}$-in duplicate amplifications- depended mainly on the starting amount of template, for both the high molecular weight samples and the degraded ones.

\subsection{Threshold for locus call}

The Coverage Analysis v5.0.4.0 plugin is set up to provide a genotype when the locus reaches a coverage $\geq 6 \mathrm{x}$. The use of a threshold for the "locus call" (e.g., its genotyping) has been debated from the very beginning of the application of PCR-MPS technologies in Forensic Genetics, and a minimum of $20 \mathrm{x}$ has been suggested as a reliable threshold [12]. To date, a wide range of thresholds, from $6 \mathrm{x}$ to $200 \mathrm{x}$, have been used in different studies characterizing the Precision ID Identity Panel [12-14,23,26-28] as well other SNP-based methods [39-41]. In the present study, the definition of this threshold has been empirically defined as follows. 
Using the PCR-MPS technology [13,15], in the $0.1-1$ ng of high molecular weight DNA input range, each locus is usually covered by hundreds of reads, whereas locus coverage drops when lower amounts of degraded templates are analyzed [26,27]. Remarkably, the frequency of PCR artefacts (mainly allele drop outs) also increases when low amounts of degraded samples are studied [27,29]. Based on these findings, in order to set up the best analytical threshold for the kind of samples selected for the present study, three different threshold values $(20 \mathrm{x}, 50 \mathrm{x}$ and $100 \mathrm{x}$, respectively) were tested and applied to the PCR-MPS data recovered from two very challenging samples (S2 and S21); to this aim, 12 pg DNA replicated analysis of the two above mentioned degraded samples and the same amount of the control DNA 2800M were set up (see Table S5). As expected, the percentage of markers with a coverage higher than the three selected thresholds decreased (from $59.9 \%$ to $31.9 \%$ for sample S2; from $56.9 \%$ to $34.9 \%$ for samples S21) as well as the percentages of markers showing PCR artefacts was lower (from $14.7 \%$ to $11.1 \%$ for sample S2; from $24.7 \%$ to $19.2 \%$ for sample S21).

When the $100 \mathrm{x}$ cut off value was adopted, the percentage of available markers in S2 and S21 was statistically reduced in comparison to the $20 \mathrm{x}$ cut off ( $p$-value of 0.0001 and 0.0139 , respectively; see Table S6). The following comparison between the $50 \mathrm{x}$ and $100 \mathrm{x}$ threshold revealed that the latter reduced statistically the frequency of available markers in S2 ( $p$-value $=0.0035)$. A reduction of the percentage of errors was found only in sample S21 when the results of the $100 \mathrm{x}$ analysis were compared with those of the $20 \mathrm{x}$ one $(p$-value $=0.0463)$. No statistically relevant differences between the thresholds were recorded for $2800 \mathrm{M}$ DNA control samples. Therefore, a threshold of $50 \mathrm{x}$ was considered an acceptable agreement between the number of available markers for genotyping and the frequency of errors such as drop outs. However, these artifacts could not be completely eliminated even introducing the $100 \mathrm{x}$ threshold (dropout frequencies were in the range of $11.1 \%, 19.2 \%$ and $7.1 \%$ in S2, S21 and in the 2800M DNA, respectively).

\subsection{Sensitivity}

The Precision ID Identity Panel kit is designed for the analysis of one nanogram of DNA through 21 PCR cycles even if additional cycles are recommended for lower amounts of template [31]. As reported in Table S7, to test the sensitivity of the assay, scalar amounts (from $1 \mathrm{ng}$ to $12 \mathrm{pg}$ ) of the 2800M DNA were amplified through 21-26 PCR cycles. Figure 1A shows that when the amount of input DNA decreased, the frequency markers with less than 50 reads of coverage increased. Specifically, when 12 pg of the control DNA were amplified, $9.4 \%$ and $28.4 \%$ of the autosomal and of Y-specific markers, respectively, showed less than 50 reads. The GQ (Genotype Quality) scores of the markers were, in average, higher in the OTQ and STQ samples (mean value 97.4 and 94.6, 
respectively) than in the MTQ ones (mean value 87.5). Interestingly, correct typing was provided by the software even for markers whose GQ was as low as 3.

Drop out phenomena were observed only when 50 pg DNA (or less) were used for the amplification, with an average of seven drop outs per sample $(\min 4$; $\max 11)$ in the 12 pg tests. These typing errors were observed even in markers whose GQ was optimal (e.g., 99), thus confirming that the GQ score provides only information on the quality of the sequencing data but not on the correctness of the genotyping [15].

\subsection{Optimization of the number of PCR cycles}

In a previously published paper reporting data on the prototype of the Precision ID Identity Panel [27], a control DNA sample was amplified down to 5 pg through 26 PCR cycles even if, in the same paper, it was speculated that 24-25 cycles could be sufficient to produce the same grade of results. However, in that study a systematic comparison was not performed between a different number of PCR cycles. Other data advise against the use of more than 25 cycles as stochastic effects are enhanced $[14,39]$.

Considering that additional PCR cycles could increase the number of artefacts when Low Copy Number DNA is typed [2,3,27], 12 pg DNA replicated amplifications through 23 and 25 cycles of five degraded samples (S2, S21, S17, S43 and S74) were compared in the present study. The results showed that the employment of 25 cycles increased the frequency of markers with more than 50 reads -in average- from $42.7 \%$ to $56.4 \%$ ( $p$-value $=0.011$ ) while the frequency of drop out remained the same $(16.8 \%$ vs. $18.8 \% ; p$-value $=0.232)$.

The data obtained from duplicate analyses of these five challenging samples were also assembled to generate eighteen consensus genotypes based on pairwise comparisons as shown in Table S8. This approach showed that the number of markers that could be typed was always higher when 25 cycles were performed $(p$-value $=0.031)$, while the mistyping error frequency was similar $(p$-value $=0.303)$ through the PCR cycles. When the data of triplicate tests were used to generate consensus genotypes, the samples amplified through 25 cycles provided a higher number of loci successfully typed without any genotyping error (see Table S8). All these results support then the choice of performing 25 PCR cycles when low quantity DNA amounts (12 pg) has to be characterized.

For higher amounts of template, 21-22 cycles and 23-24 cycles provided suitable coverages for OTQ and STQ samples, respectively (see Supplementary Figures 2-3), in agreement with previous studies [27]. However, when degraded/very degraded samples have to be characterized in those quantitative categories, it is recommended to add one-two PCR cycles (up to a maximum of 25 cycles). 


\subsection{Samples genotyping}

A total of 13,908 and 3,991 autosomal and Y specific markers showed at least 50 reads, respectively, and were thus considered suitable for genotyping (see Table S9 for details).

Figure 1B shows the frequencies of the markers displaying less than 50 reads, according to the three quantitative and the three qualitative categories defined in this study. The data obtained from samples with a DI $<5$ were in agreement with those of the 2800 M DNA control sample, for each of the three quantitative categories. As expected, to lower amounts of template corresponded to higher frequencies of markers with less than 50 reads of coverage. On the opposite side, an increase in degradation indexes corresponded to an increase in the frequency of markers with less than 50 reads of coverage, in each of the three quantitative categories. Thus, while the finding of poorly covered markers in the OTQ category with DI $<5$ was unusual, the frequency of markers with less than $50 \mathrm{x}$ of coverage increased dramatically in the MTQ category with high level of degradation. In conclusion, the use of this threshold allowed the genotyping of more than $50 \%$ and about $35 \%$ of the autosomal and Y markers, respectively, when 12 picograms of very degraded DNA were analyzed.

\subsection{Allelic drop out}

Out of the 479 allelic drop outs scored, 33, 112 and 334 occurred in OTQ, STQ and MTQ categories, respectively, with frequencies ranging from $0.02 \%$, for the category OQT and DI <5, to $18-23 \%$ when $12 \mathrm{pg}$ degraded/very degraded DNA were amplified (see Figure 2). One of the two alleles of the heterozygous genotypes was totally missing in 446 cases $(93.1 \%)$ whereas in the remaining 33 tests it showed a low coverage. The median GQ (genotype quality) scores of these 446 markers was 38, 55 and 58 in the OQT, SQT and MQT categories, respectively, with the highest values in the last two categories ( $p$-value $\leq 1.5 \times 10^{-4}$ ). These data pointed out that false homozygous genotypes could be assigned to a given locus as a consequence of acceptable/good GQ scores and without flags by the software alerting the analyst [15]. The 33 alleles showing low coverage (in average 7.1-8.8 \% of the reads of the locus) were always highlighted with the MAF (Major Allele Frequencies) flag, meaning an important allelic imbalance, and showed low GQ scores (median value=18; $\min =8.3 ; \max =20.0$ ). In conclusion, the message emerging from the data analysis is that only a very limited percentage (6.9\%) of the total cases of real drop-outs could be speculated in the present study if the genotype of the sample was not available.

Figure 3 shows the coverage of the surviving alleles, which ranged from $50 \mathrm{x}$ to $300 \mathrm{x}$ in about $86.8 \%$ of the cases, while in 13 cases $(2.9 \%$ ) it was even higher than $600 \mathrm{x}$ (up to 1,506 x). These data highlighted that there is no obvious stochastic threshold for locus call able to protect the expert from 
the occurrence of these amplification artefacts. A correlation coefficient $r^{2}=0.559$ was found between the molecular weight of the amplicons and the frequency of allelic drop outs (see Figure S6). As expected, the highest frequencies of allelic drop outs were scored for high molecular weight markers, even if it is well known that some low molecular weight loci are more prone to allelic imbalance than others [13-15], situation that can frequently turn out in the complete loss of the allele.

\subsection{Allelic drop in}

Only autosomal markers showed this kind of artefacts, while in ref. [27] they were described to occur for Y-SNPs as well. In total, 184 allelic drop-ins were scored. Out of them, 179 markers which were actually homozygous showed an additional allele instead (for example, $\mathrm{A} / \mathrm{A} \rightarrow \mathrm{A} / \mathrm{G}$ ), while in the remaining five cases the original homozygous pattern was replaced by the new one (for example, $A / A \rightarrow G / G)$. Supplementary Table 10 shows the main features of the markers affected by this artefact in the present study. In general, the median values of GQ ranged from 69 to 99 while the median values of the MAF was always high (> 76.3). In addition, about $96 \%$ of these drop ins were flagged by the Major Allele Frequency alert.

It has to be noted, however, that these drop-ins were not randomly distributed among the whole set samples, but rather clustered in two sub-sets of samples. The first is a set of eleven FFPE samples and the second originated from nine palmar swabs covering overall about $96 \%$ of these artifacts. In addition, the drop-ins occurred at the same loci of the same sample in replicate analyses, in both subsets. For this reason, minimal contamination issues in the DNA extraction step or minimal amounts of exogenous human DNA transferred to the samples were likely involved rather than spurious reads at high coverage. The remaining samples (including one $2800 \mathrm{M}$ control of $12 \mathrm{pg}$ ) showed a single drop-in in one of the replicate analyses likely originated from PCR, sequencing errors, or, alternatively, to a minimal contamination occurred in the pre-sequencing steps (target enrichment PCR and library building). In conclusion, drop-ins seem to be a quite rare event in the samples analyzed in this study.

\subsection{Analysis of the flags}

The Coverage Analysis v5.0.4.0 plugin provides four alerting flags: MAF (Major Allele Frequency), NoC (No Locus Call), PPC (Percentage of Positive Coverage) and COV (Coverage). These flags are evidenced for markers above the selected interpretation threshold, which in the present study is set to $\geq 50$ reads. The detailed analysis of these flags is reported as follows.

\subsubsection{MAF (Major Allele Frequency)}


1 A MAF flag is assigned by the analysis software to heterozygous or homozygous genotypes. In the

first case, the reads of the two alleles are unbalanced $(<35 \%$ or $>65 \%)$ while, in case of homozygous typing, the reads of the minor allele are $<10 \%$ compared to the reads of the more represented one [33].

A total of 769 MAF flags were scored for heterozygous genotypes. These flags occurred mainly in the MTQ category and in DNAs with higher degradation indexes (see Table S11), in agreement with what it is expected for PCR amplification of Low Copy Number DNA [1-3]. However, it is interesting to note that specific markers are more prone to allelic imbalance than others, as already reported $[14,15]$. In the present study, about $8.6 \%$ of these flags were recorded for the rs7520386 marker which was systematically flagged even in the $2800 \mathrm{M}$ control DNA (other markers involved are rs987640, rs1077376, rs876724, rs914165, rs891700 and rs1048871). No correlation was found with the molecular weight of the markers $\left(r^{2}=-0.208\right)$. In conclusion, no genotyping error was scored in the 769 MAF flagged heterozygous markers, independently from their GQ (median value: 53; min 3; $\max 99)$.

A total of 47 MAF flags were scored for markers genotyped as homozygous by the software instead of the correct heterozygous genotype. In 33 cases $(70.2 \%)$ the genotyping error was originated by the software which did not consider as true alleles the small residual number of reads $(<10 \%$ compared to the reads of the more represented allele). These situations have been already discussed in paragraph 3.10, whereas in the remaining 14 cases the flags originated by high noise ratio issues. The final operative consideration is that a marker typed as homozygous and highlighted with a MAF flag must be evaluated carefully since a drop out artefact cannot be ruled out. In this situation, the only possibility to clarify the real genotype is to perform a replicate test.

\subsubsection{NoC (No call)}

In total, $108 \mathrm{NoC}$ flags were assigned by the software to 83 and 25 autosomal and Y markers, respectively, meaning that it was not possible to unambiguously assign a genotype to these loci. They occurred in more than $98 \%$ of the cases in samples that showed a minimal contamination (see paragraph 3.11).

\subsubsection{PPC (Percentage of Positive Coverage)}

The software assigns this flag to a given marker if the forward and reverse sequencing strands are unbalanced $(<30 \%$ or $>70 \%$ ). In total, 389 flags were scored representing a frequency of $2.18 \%$. The autosomal markers rs436205, rs1463729, rs2111980 and rs354439 and the Y-marker rs2032595 were mostly involved, thus suggesting a locus-specific susceptibility [13-15]. No correlation with the 
amount of template was found. Genotype mistyping due to allelic drop outs was scored in 20 cases for which minimal amounts of degraded DNA was used for PCR amplification. The corresponding GQ of these 20 markers ranged from 11 to 99 (median value $=87$ ).

\subsubsection{COV (Coverage)}

The software flags a marker when its coverage is less than two standard deviations the average coverage of the sample and, according to the instruction manual [33], this flag should be able to detect potential allelic drop out artefacts. In total, $70 \mathrm{COV}$ flags were scored from the analysis of good quality samples amplified with optimal and sub-optimal amounts of template. Two autosomal (rs2342747 and rs214955) and two Y markers (rs372157627 and rs4141886) were mainly flagged, thus assuming a locus-specific predisposition [14,15]. At the end, only a single allelic drop out artefact was identified.

\subsection{Inhibited samples}

Sixteen samples (4 bones, 11 touch DNAs and 1 buccal swab) displayed low sequencing depth compared to what emerged from the sensitivity studies described above, even if a suitable amount of DNA (ranging from $90 \mathrm{pg}$ to $1 \mathrm{ng}$ ) was added for library reparation. In order to check the presence of any PCR inhibitor in these samples, new libraries were built amplifying diluted volumes (down to 1:20) of input DNA and then submitting them to sequencing runs. This approach allowed a remarkable improvement of all the sequencing parameters (see Figures S7A and S7B) as well a successful typing in 15/16 samples. In conclusion, these results strongly support the hypothesis that PCR inhibition $[42,43]$ occurred in the first round of amplifications.

\subsection{Experiments with undiluted libraries}

Three libraries displaying quantification values ranging from 21.1 to $23.0 \mathrm{pM}$ were selected in order to check if an increase in the library concentrations during the emulsion PCR step could improve the genotyping efficiency, when low-level DNA is sequenced. To this aim, replicate analyses were performed by adding either the above mentioned libraries as such or diluted to the concentration suggested by the manufacturers ( $8 \mathrm{pM}$ ) (see Table S12). The tests displayed a high level of repeatability of the $\mathrm{rDoC}\left(r^{2} \geq 0.979\right)$. As expected, the use of undiluted libraries increased the values of mapped reads, mean depth of coverage and number of markers with coverage $\geq 50 \mathrm{x}$ but, on the other hand, this caused an increment of the number of genotyping errors as well. The error frequency between the two sets of samples remained however statistically the same ( $p$-value $=0.280$ ). These 
results showed that this approach could be considered a further tool for improving the genotyping of the most challenging samples (see below).

\subsection{Experiments with additional PCR cycles after library preparation}

Six amplified libraries were submitted to an optional step of re-amplification, by adding eight extra amplification cycles (see Table S13). Libraries amplified for 23 cycles and re-amplified with additional cycles $(23+8$ cycles $)$ were loaded and sequenced in two different chips. The tests displayed a high level of repeatability $\left(r^{2} \geq 0.983\right)$ and showed an improvement of the values of the main sequencing parameters (see Figure S8) together with the number of markers with a coverage above 50 reads. Similarly, even if an increment of the number of the genotyping errors was scored, the error frequency remained statistically the same in the two sets of samples ( $p$-value $=0.332$ ). In conclusion, although further studies are needed, our results confirm that the use of eight additional cycles of amplification could be a useful tool for improving the results from low concentration libraries [31].

\subsection{Assessment of non-human DNA sequencing in bone samples}

Thirty-three libraries bam files originated from the analysis of eighteen bone samples were checked in order to verify the presence of non-human DNA sequences (see Table S14). Out of them, twenty libraries coming from eleven bone samples showed a percentage of unmapped reads above $10 \%$ and poor genotyping results, and were thus selected to be assessed for the presence of non-human DNA sequences. This analysis revealed the presence of a 50 nucleotides long sequence in all these libraries, representing up to the $10 \%$ of the unmapped reads; the following check on BLAST® detected similarity between this sequence and Mycolicibacterium smegmatis. Sample S36 revealed the presence of one group of 50 nucleotides long sequences which found similarity with Pseudomonas extremaustralis. Similarly, in sample S38 a group of 55 nucleotides long sequences was found, which revealed similarity with Lysobacter enzymogenes. These data confirmed that bacterial DNA can be efficiently ligated into the libraries and sequenced [27], and provided further data on the bacterial species which colonize skeletal remains [44]. Few samples showed a 26 nucleotides long sequence, which did not return any result on BLAST®. It is interesting to note that this $26 \mathrm{nt}$ sequence (5'CCAGAGGCTTCGGAGGAGGGCTGGCC3') was also found in no-template controls in remarkable amounts. The origin of this sequence remains unknown as it does not match either with those of the adaptamers or with other sequences used for the library construction. 


\section{Final remarks}

The data presented in this study addressed the central role of the threshold value for locus call in molecular analysis. It is well known that the minimum depth of coverage to assign a genotype depends on the sequencing technology and on the end-point of the study $[9,12]$; in forensic genetic studies based on PCR-MPS of SNPs, it widely ranged from a minimum of 6 [27,28] up to 200 reads [21,39] depending on the set of specimens considered and the frequency of errors that it was intended to accept in response to the increased number of scorable markers $[9,41]$. In this paper, the threshold for the analysis of the data was fixed for all the markers at $50 \mathrm{x}$ of coverage after having tested three different values (20 x, $50 \mathrm{x}$ and $100 \mathrm{x}$ ) on a set of LCN-DNA and degraded samples. Our evaluation was that the $50 \mathrm{x}$ threshold represented a proper balance between the two issues above mentioned, allowing to type in average, for each amplification, more than 50 and $35 \%$ of autosomal and $\mathrm{Y}$ specific markers, respectively, and to limit the number of errors when challenging samples were examined. In addition, according to the data presented in this study, the amplification for 25 PCR cycles of the Precision ID Identity Panel kit represented the most appropriate experimental condition in order to obtain a set of markers displaying valuable molecular data even from the most challenging samples. In conclusion, however, the results here reported clearly pointed out the need of setting up a consensus profile [38] when LCN-DNA and degraded samples are tested in order to minimize the risk of mistyping. This consensus approach, in fact, allowed the typing -in average- of 33 out of 90 autosomal markers (see Table S8) and provided a good statistical support to the genetic evidence. In detail, assuming 0.41 as the average random match probability (RMP) of each autosomal SNP marker [24,25], the combined RMP was of at least $1.6 \times 10^{-13}$. Thus, even if only $37 \%$ of the autosomal loci were typed, the high number of markers which can be analysed by PCR-MPS provided however a high discriminatory power that underlined the usefulness of the SNP markers in the analysis of degraded samples $[4,5,26,27,44,45]$.

A critical issue emerged from the quantification of low concentrated libraries. In fact, about $45 \%$ of the libraries built with $12 \mathrm{pg}$ of template showed picomolarities overlapping those of the no-template controls thus leading to an overestimation of the real concentrations of those samples; this caused the typing of only a limited number of markers (20-40\% of SNPs above 50 reads). In order to increase the percentages of markers above the $50 \mathrm{x}$ threshold for these LCN-DNAs, two strategies were adopted. The first considered to add eight PCR cycles to the standard 23 as suggested by the manufacturer [31] and the second one to employ undiluted, not normalised, libraries. Both approaches produced an increase in the number of markers with $\geq 50$ reads but no statistical significant increment in the error rates. Although both these approaches seem promising, they require extensive evaluation and validation before routine use in forensic genetics. 
1 A further critical issue emerged from the analysis of a set of samples (mainly bones and palmar swabs) which gave no or very poor coverage when $0.2-1 \mathrm{ng}$ of DNA (in high volumes as much as $10 \mu \mathrm{l}$ ) were tested. These samples were then diluted down to 1:20 and tested again thus providing good locus coverages with the expected number of markers above the threshold. These results led us to speculate that the Precision ID Identity Panel was sensitive to inhibition [41,42]. This is a tricky situation to prevent as these samples showed IPC values $<26 \mathrm{Cq}$, not pointing out any inhibition, when quantified with the Quantifiler ${ }^{\mathrm{TM}}$ Trio DNA Quantification Kit, which was however performed using a limited volume of DNA $(2 \mu \mathrm{l})$.

Finally, non-human DNA sequences belonging to different bacterial species were characterised analysing libraries bam files originated from bone samples buried in soil showing a percentage of unmapped reads above $10 \%$ and poor human-specific coverage. PCR-MPS technology offers the possibility to study these unmapped reads thus revealing the microorganism to which they belong. This can potentially lead to the definition of a soil microbiome profile, peculiar of that specific bone sample, which can be further compared with that of the soil where the bones were recovered or with different soil samples if there is the suspect that the bones were transferred from another burial site.

\section{References}

[1] J. M. Butler, Advanced Topics in Forensic DNA Typing: Methodology, Academic Press, Boston, 2011.

[2] P. Gill, H. Haned, O. Bleka, O. Hansson,G. Dørum, T. Egeland, Genotyping and interpretation of STR-DNA: Low template, mixtures and database matches-Twenty years of research and development. Forensic Sci. Int. Genet. 18 (2015) 100-117.

[3] J. M. Butler, M. D. Coble, P. M. Vallone, STRs vs. SNPs: thoughts on the future of forensic DNA testing, Forensic Sci. Med. Pathol. 3 (3) (2007) 200-205.

[4] B. Sobrino, M. Briòn, A. Carracedo, SNPs in forensic genetics: a review on SNP typing methodologies Forensic Science International 154 (2005) 181-194.

[5] B. Budowle, A. van Daal, Forensically relevant SNP classes, Biotechniques, 44 (5) (2008) 603608.

[6] R. Alaeddini, S.J. Walsh, A. Abbas, Forensic implications of genetic analyses from degraded DNA-a review, Forensic Sci. Int. Genet. 4 (2010), 148-157.

[7] J.G. Shewale, Forensic DNA analysis. Current practices and emerging technologies, CRC Press, Boca Raton, 2014.

[8] C. Børsting, N. Morling, Next generation sequencing and its applications in forensic genetics, Forensic Sci. Int. Genet. 18 (2015) 78-89. 
1 [9] S. Goodwin, J.D. McPherson, W.R. McCombie, Coming of age: Ten years of next generation sequencing technologies, Nat. Rev. Genet. 17 (2016) 333-351.

[10] S.B. Seo, J.L., King, D.H., Warshauer, C.P. Davis, J. Ge et al. Single nucleotide polymorphism typing with massively parallel sequencing for human identification. Int. J. Legal Med. 127 (2013) 1079-1086.

[11] C. Børsting, S. L. Fordyce, J. Olofsson, H. S. Mogensen, N. Morling, Evaluation of the Ion Torrent HID SNP 169-plex: a SNP typing assay developed for human identification by second generation sequencing, Forensic Sci. Int. Genet. 12 (2014) 144-154.

[12] M. Eduardoff, C. Santos, M. de la Puente, T. E. Gross, M. Fondevila, C. Strobl, B. Sobrino, D. Ballard, P. M. Schneider, A. Carracedo, M. V. Lareu, W. Parson, C. Phillips, Inter-laboratory evaluation of SNP-based forensic identification by massively parallel sequencing using the Ion PGMTM, Forensic Sci. Int. Genet. 17 (2015) 110-121.

[13] F. Guo, Y. Zhou, H. Song, J. Zhao, H. Shen, B. Zhao, F. Liu, X. Jiang, Next generation sequencing of SNPs using the HID-ion AmpliSeqTM identity panel on the ion torrent PGMTM platform, Forensic Sci. Int. Genet. 25 (2016) 73-84.

[14] A. Buchard, M. L. Kampmann, L. Poulsen, C. Børsting, N. Morling, ISO 17025 validation of a next-generation sequencing assay for relationship testing, Electrophoresis 37 (2016) 2822-2831.

[15] K. A. Meiklejohn, J. M. Robertson, Evaluation of the Precision ID Identity Panel for the Ion Torrent ${ }^{\mathrm{TM}} \mathrm{PGM}^{\mathrm{TM}}$ sequencer, Forensic Sci. Int. Genet. 31 (2017) 48-56.

[16] O. Garcia, A. Soto, I. Yurrebaso, Allele frequencies and other forensic parameters of the HIDIon AmpliSeq Identity Panel markers in Basques using the Ion Torrent PGM platform, Forensic Sci. Int. Genet. 28 (2017) e8-e10.

[17] R. Li, C. Zhang, H. Li, R. Wu, H. Li, Z. Tang, C. Zhen, J. Ge, D. Peng, Y. Wang, H. Chen, H. Sun, SNP typing using the HID-Ion AmpliSeq Identity Panel in a southern Chinese population, Int. J. Legal Med. 132 (2017) 997-1006.

[19] S. van der Heijden, S. Juel de Oliveira, M. L. Kampmann, C. Børsting, N. Morling, Comparison of manual and automated AmpliSeq ${ }^{\mathrm{TM}}$ workflows in the typing of a Somali population with the Precision ID Identity Panel, Forensic Sci. Int. Genet. 31 (2017) 118-125.

[20] J. Liu, Z. Wang, G. He, X. Zhao, M. Wang, T. Luo, C. Li, Y. Hou, Massively parallel sequencing of 124 SNPs included in the precision ID identity panel in three East Asian minority ethnicities, Forensic Sci. Int. Genet. 35 (2018) 141-148.

[21] M.-L. Kampmann, A. Buchard, C. Børsting, N. Morling, High-throughput sequencing of forensic genetic samples using punches of FTA cards with buccal swabs, Biotechniques 61 (2016) 149-151. 
[22] L. Sun, L. Fu, Q. Liu, J. Zhou, C. Ma, B. Cong, S. Li, Population data using Precision ID Identity Panel in a Chinese Han population from Hebei Province, Forensic Sci. Int. Genet. 4 (2019) e27-e29. [23] S. L. Christiansen, B. Jakobsen, C. Børsting, H. Udengaard, A. Buchard, M. L. Kampmann, M. Grøndahl, N. Morling, Non-invasive prenatal paternity testing using a standard forensic genetic massively parallel sequencing assay for amplification of human identification SNPs, Int. J. Legal Med. 133 (2019) 1361-1368.

[24] E. Huang, C. Liu, J. Zheng, X. Han, W. Du, Y. Huang, C. Li, X. Wang, D. Tong, X. Ou, H. Sun, Z. Zeng, C. Liu, Genome-wide screen for universal individual identification SNPs based on the HapMap and 1000 Genomes databases, Sci. Rep. 8 (2018) 5553.

[25] P.H. Sudmant, T. Rausch, E.J. Gardner, R.R. Handsaker, A. Abyzov, J. Huddleston et al., An integrated map of structural variation in 2504 genomes, Nature 526 (2015) 75-81.

[26] K. B. Gettings, K. M. Kiesler, P. M. Vallone, Performance of a next generation sequencing SNP assay on degraded DNA, Forensic Sci. Int. Genet. 19 (2015) 1-9.

[27] E. Salata, A. Agostino, I. Ciuna, S. Wootton, L. Ripani, A. Berti, Revealing the challenges of low template DNA analysis with the prototype Ion AmpliSeq ${ }^{\mathrm{TM}}$ Identity panel v2.3 on the PGMTM Sequencer, Forensic Sci. Int. Genet. 22 (2016) 25-36.

[28] E. Avila, C. P. Cavalheiro, A. B. Felkl, P. Graebin, A. Kahmann, C. S. Alho, Brazilian forensic casework analysis through MPS applications: Statistical weight-of-evidence and biological nature of criminal samples as an influence factor in quality metrics. Forensic Sci. Int. 303 (2019) 109938.

[29] P. Fattorini, C. Previderè, I. Carboni, G. Marrubini, S. Sorçaburu-Cigliero et al., Performance of the ForenSeqTM DNA Signature Prep kit on highly degraded samples, Electrophoresis 38 (2017) $1163-1174$.

[30] P. Fattorini, C. Previderè, S. Sorçaburu-Cigliero, G. Marrubini, M. Alù et al., The molecular characterization of a depurinated trial DNA sample can be a model to understand the reliability of the results in forensic genetics, Electrophoresis 35 (2014) 3134-3145.

[31] Thermo Fisher Scientific, Precision ID Panels with the Ion PGM ${ }^{\mathrm{TM}}$ System, Application Guide, MAN0015830, Revision B.0, 2017.

[32] C. Hussing, M. L. Kampmann, H. Smidt Mogensen, C. Børsting, N. Morling, Quantification of massively parallel sequencing libraries - a comparative study of eight methods, Sci. Rep. 8 (2018) 1110.

[33] Thermo Fisher Scientific, HID SNP Genotyper Plugin User Guide v4.3.2, MAN0010641, Revision C.0, 2016.

[34] https://usegalaxy.org/

[35] http://www.bioinformatics.babraham.ac.uk/projects/fastqc/ 
[36] https://blast.ncbi.nlm.nih.gov/Blast.cgi

[37] J.D. Churchill, S.E. Schmedes, J.L. King, B. Budowle, Evaluation of the Illumina(®) Beta Version ForenSeq ${ }^{\mathrm{TM}}$ DNA Signature Prep Kit for use in genetic profiling, Forensic Sci. Int. Genet. 20 (2016) 20-29.

[38] P. Taberlet, S. Griffin, B. Goossens, S. Questiau, S. Manceau et al., Reliable genotyping of samples with very low DNA quantities using PCR, Nucleic Acids Res. 24 (1996) 3189-3194.

[39] V. Pereira, H. S. Mogensen, C. Børsting, N. Morling, Evaluation of the Precision ID Ancestry Panel for crime case work: A SNP typing assay developed for typing of 165 ancestral informative markers, Forensic Sci. Int. Genet. 28 (2017) 138-145.

[40] D. L. T. Apaga, S. E. Dennis, J. M. Salvador, G. C. Calacal, M. C. A. De Ungria, Comparison of Two Massively Parallel Sequencing Platforms using 83 Single Nucleotide Polymorphisms for Human Identification, Sci. Rep. 7 (2017) 398.

[41] K. Song, L. Li. G. Zhang, Coverage recommendation for genotyping analysis of highly heterologous species using next generation sequencing technology, Sci. Rep. 6 (2016) 35736. [42] K. Elwick, X. Zeng, J. King, B. Budowle, S. Hughes-Stamm, Comparative tolerance of two massively parallel sequencing systems to common PCR inhibitors, Int. J. Legal Med. 132 (4) (2018)983-995.

[43] M. Sidstedt, C.R. Steffen, K.M. Kiesler, P.M. Vallone, P. Rådström, J. Hedman, The impact of common PCR inhibitors on forensic MPS analysis, Forensic Sci. Int. Genet. 40 (2019) 182-191.

[44] A. Kazarina, G. Gerhards, E. Petersone-Gordina, J. Kimsis, I. Pole et al., Analysis of the bacterial communities in ancient human bones and burial soil samples: Tracing the impact of environmental bacteria, J. Archaeol. Scie. 109 (2019) 104989.

[45] A. Tillmar, I. Grandell, K. Montelius, DNA identification of compromised samples with massive parallel sequencing, Forensic Sci. Res. 18 (2018) 1-7.

[46] E. I. Zavala, S. Rajagopal, G.H. Perry, I. Kruzic, Ž. Bašić et al., Impact of DNA degradation on massively parallel sequencing-based autosomal STR, iiSNP, and mitochondrial DNA typing systems, Int. J. Legal Med. 133(5) (2019) 1369-1380. 
8

\begin{tabular}{|l|c|c|c|c|}
\hline & $\begin{array}{c}\text { OTQ } \\
(\mathbf{0 . 1 - 1 . 0 ~} \mathbf{~ n g})\end{array}$ & $\begin{array}{c}\text { STQ } \\
(\mathbf{1 3 - 9 9} \mathbf{~ p g})\end{array}$ & $\begin{array}{c}\text { MTQ } \\
(\mathbf{1 2} \mathbf{~ p g})\end{array}$ & NT-CTRL \\
\hline$n$ & 92 & 50 & 51 & 17 \\
\hline Library concentration (pM) & 132.4 & 57.8 & 20.3 & 9.3 \\
\hline Mapped reads & 70,929 & 52,374 & 23,475 & 4,415 \\
\hline Mean depth of coverage & 494.5 & 252.7 & 121.3 & 0.8 \\
\hline \% of on-target reads & 94.0 & 85.9 & 76.4 & 7.7 \\
\hline
\end{tabular}

9 Table 1. Pooled main sequencing parameters of the samples analysed in this study according to the three quantitative categories OTQ, STQ and MTQ, respectively (the values refer to median values). NT-CTRL: no template control; $n=$ number of tests. 


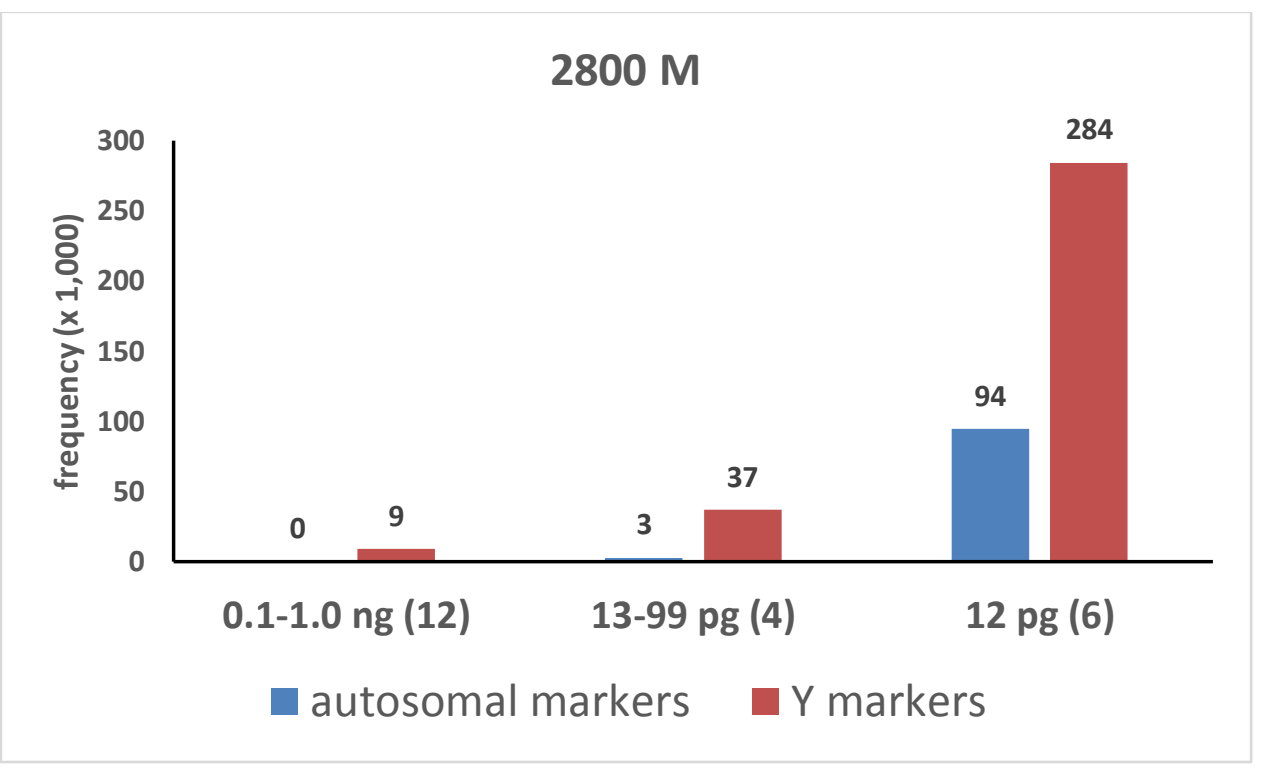

4 Figure 1A. Frequencies of markers showing less than $50 \mathrm{x}$ of coverage in the $2800 \mathrm{M}$ cell line DNA.

5 The results are pooled according to each of the three quantitative categories based on the amount of 6 input DNA (in brackets the number of tests). Y-axis: frequency (x 1,000).

7

8

9

10

11

12

13

14

15

16

17

18 


\section{OTQ (0.1-1.0 ng of DNA)}

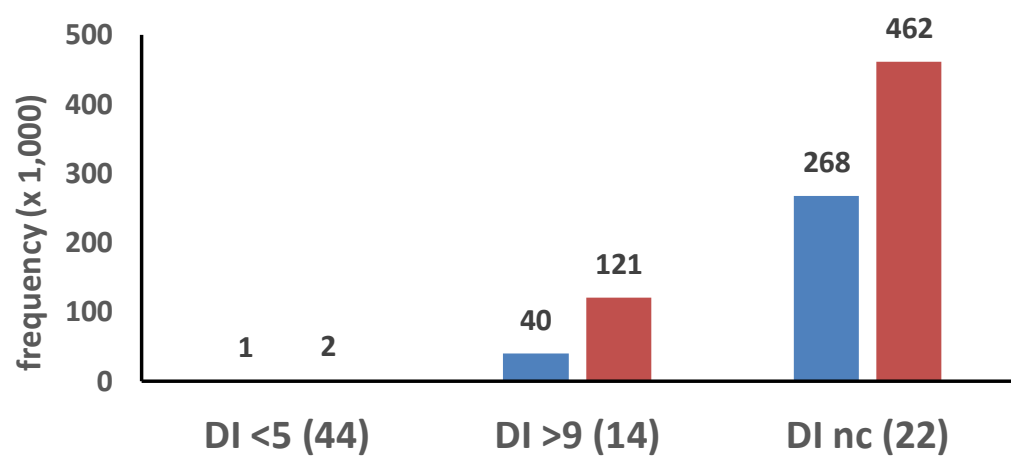

autosomal markers $\quad$ Y markers

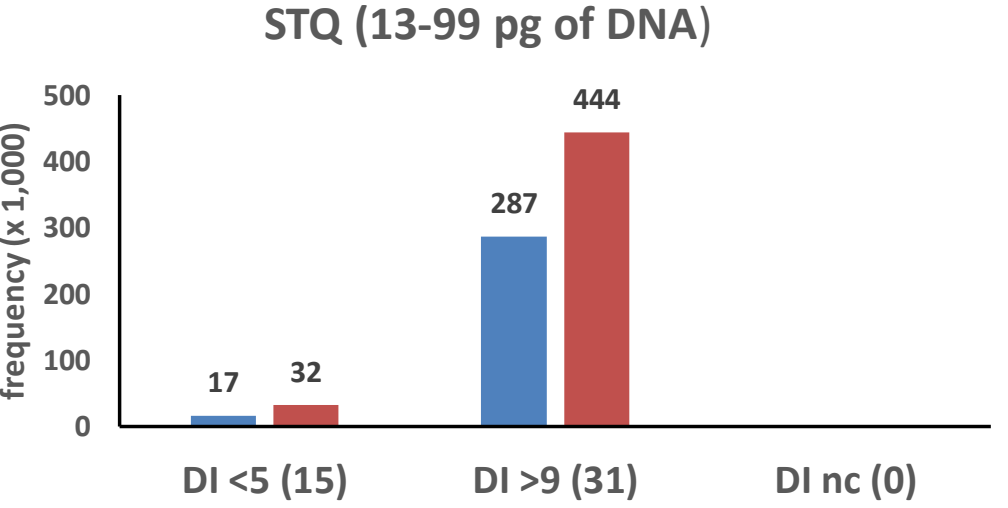

2

autosomal markers $\square$ Y markers

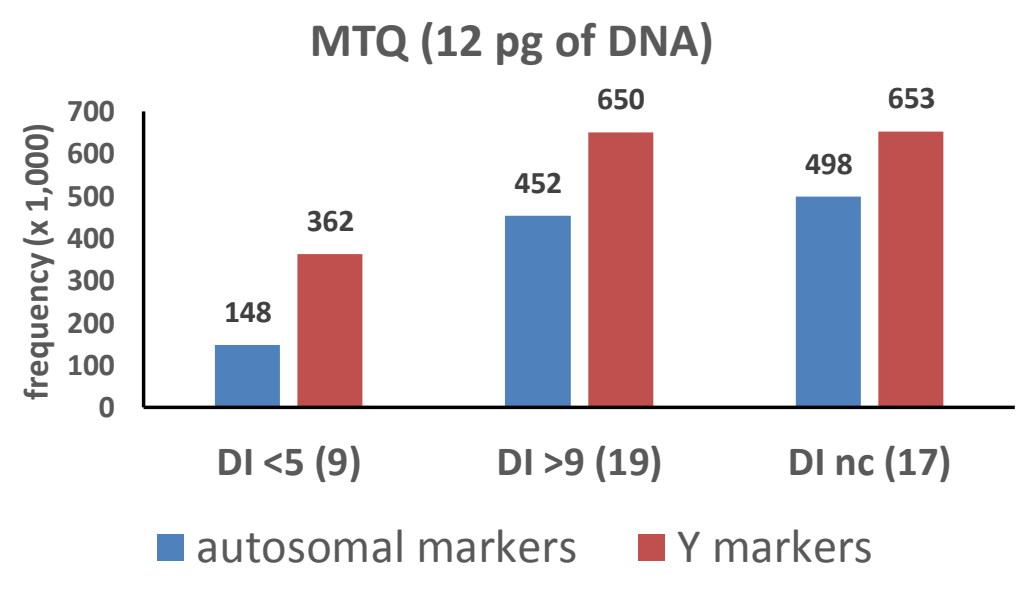

4 Figure 1B. Frequencies of markers showing less than $50 \mathrm{x}$ of coverage in the samples employed in

5 this study. The results are pooled according to the nine classes obtained from the combination of the

6 three quantitative (OTQ, STQ, MTQ) and the three qualitative categories (based on the DI). In

7 brackets the number of tests. Y-axis: frequency (x 1,000). 


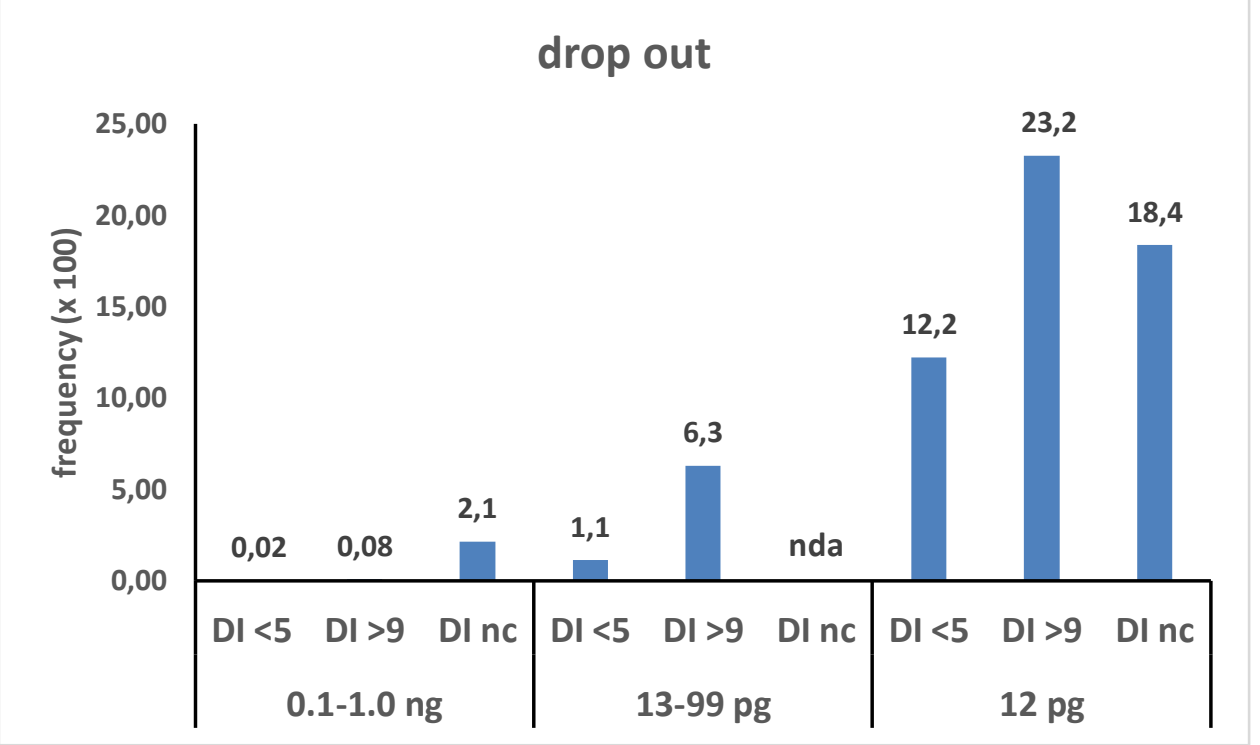

2 Figure 2. Percentage frequencies of allelic drop outs. The results are pooled according to the nine 3 classes obtained from the combination of the three quantitative (0.1-1.0 ng, 13-99 pg and $12 \mathrm{pg}$ ) and 4 the three qualitative categories (based on the DI). Y-axis: frequency (x 100). nda: no data available. 


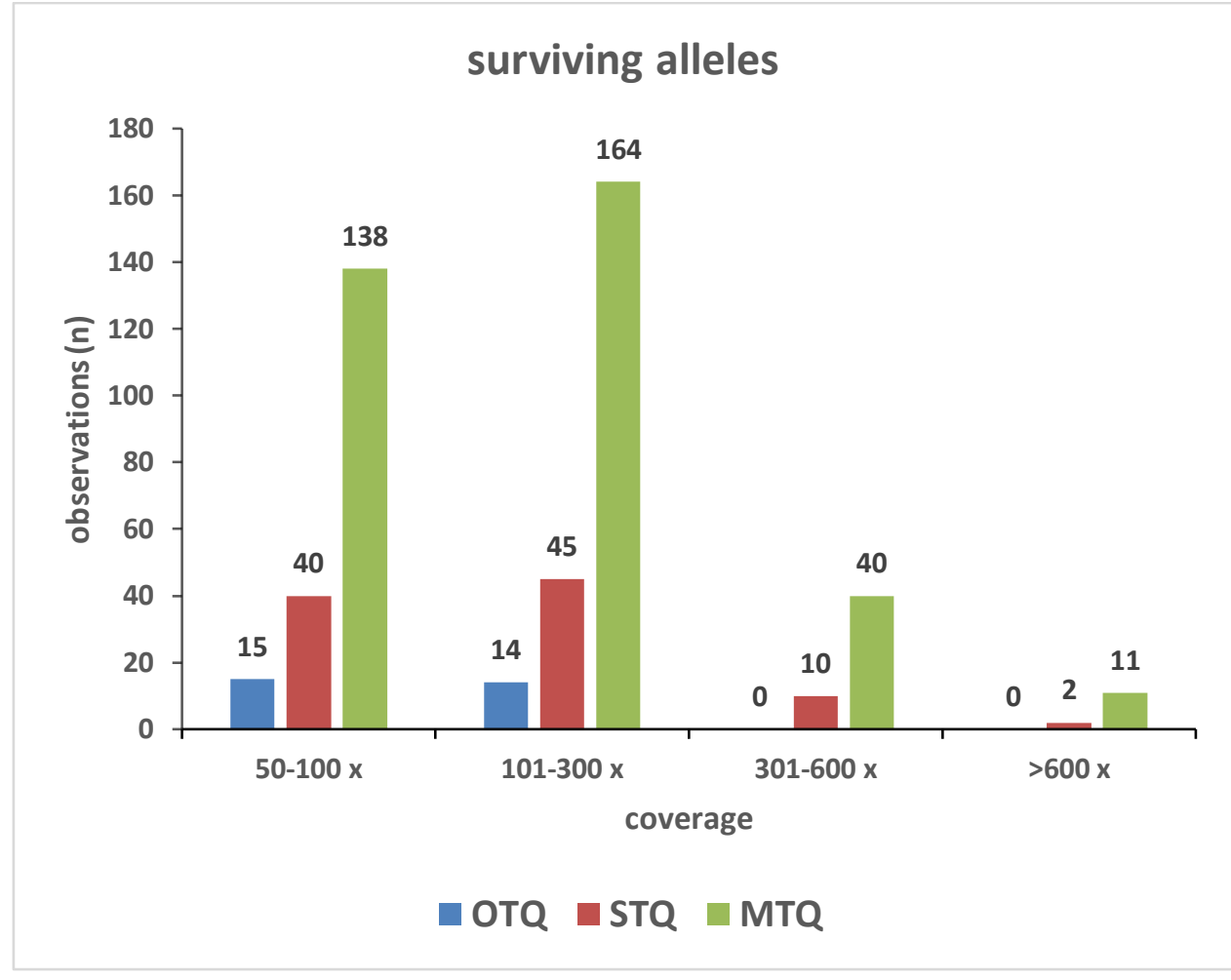

2 Figure 3. Coverage of the 479 surviving alleles scored in this study, according to the three 3 quantitative categories (OTQ, STQ, MTQ). 